\title{
The Influence of Family History on Stage and Survival of Gastric Cancer According to the TGFB1 C-509T Polymorphism in Korea
}

\author{
Hee Jin Kim ${ }^{1,2}$, Mingu Kwon ${ }^{1}$, Nayoung Kim ${ }^{1,3,4}$, Jae Bong Lee ${ }^{5}$, and Sungho Won ${ }^{6}$ \\ ${ }^{1}$ Department of Internal Medicine, Seoul National University Bundang Hospital, Seongnam, ${ }^{2}$ Department of Internal Medicine, Myongji \\ Hospital, Hanyang University College of Medicine, Goyang, ${ }^{3}$ Department of Internal Medicine and Liver Research Institute, and ${ }^{4}$ Tumor \\ Microenvironment Global Core Research Center, Seoul National University, Seoul, ${ }^{5}$ Division of Statistics, Medical Research Collaborating \\ Center, Seoul National University Bundang Hospital, Seongnam, and ${ }^{6}$ Department of Public Health Sciences, Seoul National University, Seoul, \\ Korea
}

Background/Aims: The survival rate of gastric cancer (GC) is known to be higher in patients with a family history (FH) of GC. There is an association between a polymorphism in the transforming growth factor- $\beta 1$ (TGFB1) gene and the risk of GC in patients with first-degree relatives with GC. This study was performed to investigate whether a FH affects GC outcomes according to the TGFB1 C-509T polymorphism. Methods: TGFB1 was genotyped by the polymerase chain reaction-restriction fragment length polymorphism method in 1,143 GC patients, including 216 patients (18.9\%) with firstdegree relatives with GC. Results: The proportion of stage I-II GCs was significantly higher in patients with a FH than in those without a FH of GC (83.8 vs $74.9 \%, p=0.005)$. The association between a FH of GC and stage I-II GC was not significant in subgroups divided based on the TGFB1 C-509T polymorphism and sex. A FH did not affect the overall survival rate of GC in patient with all stages and each stage. The overall survival rates were not significantly different between patients with the $\mathrm{CC}$ and $\mathrm{CT} / \mathrm{TT}$ genotypes of the TGFB1509 polymorphism. Conclusions: Patient with a FH of GC had lower cancer stage (I-II) at diagnosis than those without a FH of GC, but there was no significant difference in overall survival between the patients with and without a FH of GC. A FH did not influence the tumor stage or overall survival in patients stratified by the presence of the TGFB1 C-509T polymorphism. (Gut Liver 2020;14:79-88)

Key Words: Transforming growth factor beta1; Polymorphism; Gastric cancer; Family medical history; Sex

\section{INTRODUCTION}

Despite the decreasing incidence and mortality of gastric cancer (GC) in recent decades worldwide, GC ranks as the fifth most common cancer in incidence and the third most common cause of death from cancer worldwide. ${ }^{1}$ Gastric carcinogenesis is a multistep and a multifactorial process in which Helicobacter pylori infection plays a pivotal role, ${ }^{2}$ and complex interactions between genetic and environmental factors are involved. Smoking, high consumption of salted and nitrated food, low socioeconomic status and heavy alcohol consumption are known to be independent risk factors for GC. ${ }^{3,4}$ Genetic alternations, such as mutations, single nucleotide polymorphisms, and DNA methylation, are associated with the development of GC. ${ }^{5}$

A family history (FH) of GC is also a strong risk factor for GC. ${ }^{6}$ Most GCs are sporadic, and approximately $10 \%$ of GC shows familial clustering; however, only approximately $1 \%$ to 3\% of GCs comes from inherited GC predisposition syndromes, such as hereditary diffuse gastric carcinoma and familial adenomatous polyposis. ${ }^{7}$ The risk of GC in people with a FH is approximately 3 -fold higher than in those without a FH. ${ }^{6}$ There are a limited number of studies on the association of a $\mathrm{FH}$ with the survival of GC patients. ${ }^{8-11}$ A meta-analysis of five studies reported the beneficial effects of a FH on the survival of GC..$^{12}$ It is not clear why a FH of GC affects patient survival. People with a FH tend to undertake health screenings early and frequently, ${ }^{13}$ and they usually show good health-related behaviors, such as nonsmoking, nonalcoholic drinking, and consistent exercise. In addition, genetic differences, such as microsatellite instability, ${ }^{8}$ in FHs may be associated with a good prognosis.

\footnotetext{
Correspondence to: Nayoung Kim

Department of Internal Medicine, Seoul National University Bundang Hospital, 82 Gumi-ro 173beon-gil, Bundang-gu, Seongnam 13620, Korea

Tel: +82-31-787-7008, Fax: +82-31-787-4051, E-mail: nayoungkim49@empas.com

Received on October 18, 2018. Revised on February 26, 2019. Accepted on March 23, 2019. Published online January 7, 2020.

pISSN 1976-2283 eISSN 2005-1212 https://doi.org/10.5009/gnl18471

Hee Jin Kim and Mingu Kwon contributed equally to this work as first authors.

@. This is an Open Access article distributed under the terms of the Creative Commons Attribution Non-Commercial License (http://creativecommons.org/licenses/by-nc/4.0) which permits unrestricted non-commercial use, distribution, and reproduction in any medium, provided the original work is properly cited.
} 
Transforming growth factor-beta (TGF- $\beta$ ) is known to have a dual role of inhibiting and promoting carcinogenesis; TGF- $\beta$ suppresses the proliferation of normal epithelial and lowinvasive cancer cells but enhances the proliferation of highly invasive cancer cells by stimulating angiogenesis and suppressing the immune response. The TGFB1 C-509T polymorphism, which is in the promoter region of the TGFB1, has been found to directly influence its gene expression and is most commonly studied in elucidating its association with the risk of various cancers. ${ }^{14}$ For GC, it can be concluded that the TGFB1-509T allele is susceptible for GC, although there is inconsistency among studies. ${ }^{15-18}$ Our research group have reported that the CT genotype in the TGFB1-509 polymorphism was associated with an increased risk in the development of GC (odds ratio [OR], 1.35; 95\% confidence interval [CI], 1.07 to 1.71), especially for intestinal-type GC (OR, 1.43; 95\% CI, 1.08 to 1.90$).{ }^{16}$ In addition, we reported a study of 123 GC patients with a FH and 639 age- and sex-matched control GC patients without a FH; in that study, we found that intestinal-type GC patients with a FH spent their youth in rural areas more frequently (OR, 2.0; 95\% CI, 1.0 to 3.9), and had fewer TGFB1-509T (OR, 0.5; 95\% CI, 0.3 to 0.9) than GC patients without a FH. ${ }^{19}$ Ebert et al..$^{20}$ reported increased TGF- $\beta 1$ expressions in the gastric mucosa of patients with GC and their first-degree relatives (FDRs) compared with healthy controls. From these results, it is suggested that both genetic polymorphisms and environmental factors affect the carcinogenesis of intestinal-type GC in patients with a FH. Furthermore, we assumed that the CC genotype in TGFB1-509 polymorphism might be involved in a better prognosis in patients with a FH of GC.

The incidence rate of GC was generally 2- to 3- folds higher in males than females, ${ }^{1}$ and the disparity in the survival between sexes has not been fully evaluated. A recent Korean study showed that female GC patients were significantly younger and associated with signet ring cell carcinoma compared with male patients, and had a significantly poorer overall survival, especially among young patients (aged $\leq 45$ years) with advanced $\mathrm{GC}^{21}$ It was concluded that female is a significantly poorer prognostic factor among young patients with signet ring cell carcinoma. Our previous research demonstrated that the effect of obesity on GC showed a sex difference; that is, obesity was related to an increased risk of early GC and well- or moderately differentiated adenocarcinoma in males but not in females. ${ }^{22}$ Gastric carcinogenesis appears to differ according to sex. This difference has been explained by environmental factors, including $H$. pylori infection, smoking, and dietary patterns, and hormones such as estrogen. ${ }^{13,23}$

On the basis of these published findings and the distinct roles of TGF- $\beta 1$, our hypothesis is that a FH of GC would affect the TNM stage of GC, and this influence might be associated with the CC genotype of TGFB1-509 polymorphism. To prove this hypothesis, the aim of this study was to investigate the effect of a FH on the TNM stage and overall survival according to the TGFB1 C-509T polymorphism.

\section{MATERIALS AND METHODS}

\section{Patients}

Between January 2006 and March 2017, 1,228 patients diagnosed with GC by endoscopic examinations were enrolled at the Seoul National University Bundang Hospital, South Korea. All patients were ethnically Koreans. Eighty-five patients were excluded if they met more than one of the following criteria: (1) not GC on final endoscopic or surgical pathology; (2) carcinoma in situ on final endoscopic or surgical pathology; (3) incomplete medical records; (4) TGFB1 genotyping not measured. Eventually, 1,143 GC patients were included. This study protocol was approved by the Ethics Committee at the Seoul National University Bundang Hospital (IRB number: B-1805/471-306).

The gastric mucosa from endoscopic biopsy specimens were examined for histological evaluation, determination of the $H$. pylori infection status and TGFB1 genotyping. The informed consent was provided to all patients, and they were asked to complete a questionnaire under the supervision of a trained interviewer. The questionnaire included questions regarding age, sex, smoking and drinking habits, history of $H$. pylori eradication, and FH of GC. A "positive family history" was defined as having any FDRs (parent, sibling, or offspring) diagnosed with GC. Clinicopathological data, including final pathological reports and results of computed tomography, were collected using the electronic medical chart system. GCs were staged using the 7th edition of the TNM staging system of the American Joint Committee on Cancer (2010) based on final pathologic examination. Clinical outcomes, such as recurrence or death, were obtained from medical records until the date of death, loss-tofollow-up or March 2017 (end date of the study). The causes of death were ascertained based on medical records and death certificates. Some data on date and causes of death were collected from the Ministry of Public Administration and Security and the National Statistical Office. Overall survival was calculated only in patients who underwent endoscopic and surgical resections with a curative intention and was defined as the time from diagnosis to death resulting from any cause.

\section{H. pylori infection status}

The H. pylori infection status was determined by histologic examination with rapid urease test (CLOtest; Delta West, Bentley, Australia), the Giemsa staining and culture. ${ }^{16}$ If all the results of these tests were negative, ${ }^{13} \mathrm{C}$-urea breath test (UBiTkit; Otsuka Pharmaceutical, Tokyo, Japan) and serum immunoglobulin $\mathrm{G}$ antibody for $H$. pylori detected by an enzymelinked immunosorbent assay (Genedia H. pylori ELISA; Green Cross Medical Science Corp., Eumseong, Korea) were performed. Negative $H$. pylori infection was defined when all above clinical 
tests for $H$. pylori were negative and patients did not have a history of prior $H$. pylori eradication.

\section{TGFB1 genotyping}

Genomic DNA was extracted from gastric antral mucosa by proteinase $\mathrm{K}$ digestion and phenol/chloroform extraction. The purified DNA was used to determine the genotypes of TGFB1509 using a modified method of polymerase chain reactionrestriction fragment length polymorphism (PCR-RFLP). PCR primers were as followed: 5'-GTA TGG GGT CGC AGG GTG TT-3' (forward) and 5'-CAG ATG CGC TGT GGC TTT GC-3' (reverse). The DNA was initially denatured at $95^{\circ} \mathrm{C}$ for 5 minutes, followed by 40 cycles of 30 seconds at $95^{\circ} \mathrm{C}, 30$ seconds at $62^{\circ} \mathrm{C}$, and 30 seconds at $72^{\circ} \mathrm{C}$, and the PCR was finished by a final extension cycle at $72^{\circ} \mathrm{C}$ for 10 minutes. The overnight digestion of the PCR products was performed at $37^{\circ} \mathrm{C}$ with restriction enzyme, Bsu36I (add bovine serum albumin) for TGFB1-509 genotypes (New England BioLabs, Beverly, MA, USA). The DNA fragments were separated on a vertical 2.5\% agarose gel and stained with ethidium bromide at $120 \mathrm{~V}$ for 45 minutes. The laboratory personnel were blinded to group status.

\section{Statistical analysis}

Comparisons of demographic and clinicopathologic variables were performed using the Student t-test or chi-square test (Fisher exact test) for continuous variables and categorical variables, respectively. Separate analyses were performed depending on having a FH (with and without a FH) in total, male and female groups. Survival curves were constructed using the KaplanMeier method and compared using the log-rank test. Multivariate analyses for survival were performed using the Cox proportional hazard model. All analyses were performed using SPSS for Windows, version 22 (IBM Corp., Armonk, NY, USA). The pvalues of $<0.05$ were considered statistically significant.

\section{RESULTS}

\section{Clinicopathologic characteristics of patients with GC ac- cording to a FH}

Among the 1,143 patients with GC, 216 (18.9\%) had a FH of GC. Table 1 demonstrates the clinicopathologic characteristics of patients. The female proportion was 32.4\% $(370 / 1,143)$. There were no significant differences in age, sex, smoking and drinking, body mass index, blood type, and $H$. pylori infection status between patients with and without a FH in total, male and female groups. The age at diagnosis of GC was not significantly different between patients with and without a FH in all groups. Intestinal-type GCs were not significantly more frequent in patients with a $\mathrm{FH}$ than in those without a $\mathrm{FH}$, and there were no statistically significant differences in differentiation, tumor depth and nodal metastasis between patients with and without a FH. In the total patient group, the proportion of distant metas- tasis was significantly lower in patients with a FH than without a FH (6.5\% vs $11.5 \%, p=0.029)$. In the total and male groups, the proportion of stage I-II tumors was higher in patients with a FH compared to patients without a FH (total group, 83.8\% vs $74.9 \%, p=0.005$; male group, $83.9 \%$ vs $75.3 \%$, $p=0.026$; female group, $86.3 \%$ vs $73.9 \%, \mathrm{p}=0.096)$.

\section{Association between a FH of GC and the TGFB1 C-509T polymorphism}

The genotype frequency of TGFB1-509 was not significantly different between patients with and without a FH in the total, male and female groups (Table 1). When CT and TT genotypes were combined because those were assumed to have a dominant allele effect, the frequency of T-carriers was also not significantly different between patients with and without a FH of GC (total group, 70.8\% vs 85.5\%, $\mathrm{p}=0.092$; male group, $73.2 \%$ vs $76.0 \%, p=0.475$; female group, $65.7 \%$ vs $74.6 \%, p=0.137$ ). No significant associations between polymorphism and the tumor stage in GC patients according to sex and a FH were observed (Table 2).

\section{Overall survival of GC according to a FH and the TGFB1 C-509T polymorphism}

During the follow-up period, 304 patients (26.6\%) died, and 39 patients (3.4\%) had recurrence of GC. The mean follow-up period was $86.28 \pm 46.02$ months (range, 12 to 180 months). In the female group, death was significantly lower in patients with a FH than in those without a FH, though the number of deaths was small (13.4\% vs $26.4 \%, p=0.025)$. A total of $1,030 \mathrm{GC}$ patients underwent endoscopic or surgical resection with a curative intention.

The overall survival rates for GC patients were compared according to a FH and TGFB1 C-509T polymorphism in total, male and female groups. In the female group, the overall survival rate was better in GC patients with a FH than in those without a FH, but there was no significant difference $(\mathrm{p}=0.051)$. In total and male groups, the overall survival rate was not significantly different between patients with and without a FH (total group, $p=0.596$; male group, $p=0.457)$. The overall survival rates were not significantly different between $\mathrm{CC}$ and $\mathrm{CT} / \mathrm{TT}$ genotypes of TGFB1-509 in total, male, and female groups (total group, $p=0.341$; male group, $p=0.204$, and female group, $p=0.769$ ).

Next, the overall survival rates were compared in each stage of GC. Fig. 1 shows the overall survival curves according to GC stage in patients with and without a FH. There was no significant difference in the overall survival rates between patients with and without a FH in all stages and each stage (Fig. 2). The overall survival rates were not significantly different between CC and CT/TT genotypes of TGFB1-509 in all stages, stage I-II and stage III-IV (Fig. 3). Finally, in subgroup analysis according to a $\mathrm{FH}$ and stage of $\mathrm{GC}$, the differences in the overall survival rates were not significant between $\mathrm{CC}$ and $\mathrm{CT} / \mathrm{TT}$ genotypes in 
Table 1. Clinicopathologic Characteristics of Patients Stratified by Sex and Family History of Gastric Cancer in First-Degree Relatives

\begin{tabular}{|c|c|c|c|c|c|c|c|c|c|}
\hline & \multicolumn{3}{|c|}{ Total } & \multicolumn{3}{|c|}{ Male } & \multicolumn{3}{|c|}{ Female } \\
\hline & $\begin{array}{c}\text { FH (+) } \\
(n=216)\end{array}$ & $\begin{array}{c}\text { FH (-) } \\
(n=927)\end{array}$ & p-value & $\begin{array}{c}\text { FH (+) } \\
(n=149)\end{array}$ & $\begin{array}{c}\text { FH (-) } \\
(n=624)\end{array}$ & p-value & $\begin{array}{l}\mathrm{FH}(+) \\
(\mathrm{n}=67)\end{array}$ & $\begin{array}{c}\text { FH (-) } \\
(n=303)\end{array}$ & p-value \\
\hline Age, yr & $60.42 \pm 10.36$ & $60.04 \pm 12.42$ & 0.642 & $60.93 \pm 10.12$ & $60.83 \pm 11.41$ & 0.914 & $59.27 \pm 10.87$ & $58.41 \pm 14.18$ & 0.581 \\
\hline$<40$ & 8 (3.7) & $53(5.7)$ & 0.440 & $3(2.0)$ & $19(3.0)$ & 0.763 & $5(7.5)$ & 34 (11.2) & 0.592 \\
\hline$\geq 40 \&<60$ & 89 (41.2) & 358 (38.6) & & $60(40.3)$ & 241 (38.6) & & 29 (43.3) & 117 (38.6) & \\
\hline$\geq 60$ & $119(55.1)$ & 516 (55.7) & & 86 (57.7) & 364 (58.3) & & 33 (49.3) & $152(50.2)$ & \\
\hline Sex & & & 0.637 & & & & & & \\
\hline Male & 149 (67.3) & $624(69.0)$ & & & & & & & \\
\hline Female & 67 (32.7) & 303 (31.0) & & & & & & & \\
\hline Smoking* & & & 0.620 & & & 0.507 & & & 0.383 \\
\hline Nonsmoker & $82(38.1)$ & 324 (36.3) & & $23(15.4)$ & 81 (13.3) & & $59(89.4)$ & 243 (85.3) & \\
\hline Drinking* & & & 0.984 & & & 0.776 & & & 0.548 \\
\hline Drinker & 152 (70.7) & 630 (70.6) & & 125 (83.9) & 500 (82.9) & & 27 (40.9) & $130(45.0)$ & \\
\hline Nondrinker & 63 (29.3) & $262(29.4)$ & & $24(16.1)$ & 103 (17.1) & & $39(59.1)$ & $159(55.0)$ & \\
\hline BMI, $\mathrm{kg} / \mathrm{m}^{2 *}$ & $23.37 \pm 3.21$ & $23.11 \pm 3.13$ & 0.283 & $23.49 \pm 2.88$ & $23.21 \pm 2.94$ & 0.305 & $23.11 \pm 3.85$ & $22.90 \pm 3.49$ & 0.667 \\
\hline$<23$ & 87 (42.6) & $380(45.4)$ & 0.631 & $53(38.1)$ & $234(41.5)$ & 0.635 & 34 (52.3) & 146 (53.5) & 0.967 \\
\hline$\geq 23 \&<25$ & 63 (30.9) & $231(27.6)$ & & $48(34.5)$ & $172(30.5)$ & & $15(23.1)$ & 59 (21.6) & \\
\hline$\geq 25$ & $54(26.5)$ & $226(27.0)$ & & 38 (27.3) & $158(28.0)$ & & 16 (24.6) & 68 (24.9) & \\
\hline Blood type & & & 0.659 & & & 0.450 & & & 0.723 \\
\hline B blood & 46 (21.3) & $185(20.0)$ & & $34(22.8)$ & $125(20.0)$ & & 12 (17.9) & 60 (19.8) & \\
\hline Non-B blood & 170 (78.7.3) & 742 (80.0) & & 115 (77.2) & $499(80.0)$ & & $55(82.1)$ & $243(80.2)$ & \\
\hline Positive & 187 (86.6) & 775 (83.6) & & $125(83.9)$ & $519(83.2)$ & & $62(92.5)$ & $256(84.5)$ & \\
\hline Negative & $29(13.4))$ & $152(16.4)$ & & $24(16.1)$ & $105(16.8)$ & & $5(7.5)$ & $47(15.5)$ & \\
\hline Lauren histotype & & & 0.409 & & & 0.744 & & & 0.409 \\
\hline Intestinal & $132(61.1)$ & $538(58.0)$ & & $100(67.1)$ & $410(65.7)$ & & $32(47.8)$ & $128(42.2)$ & \\
\hline Diffuse or mixed & 84 (38.9) & $389(42)$ & & 49 (32.9) & $214(34.3)$ & & $35(52.2)$ & $175(57.8)$ & \\
\hline Differentiation & & & 0.448 & & & 0.635 & & & 0.598 \\
\hline Differentiated $^{\dagger}$ & $128(59.3)$ & $523(56.4)$ & & $96(64.4)$ & $389(62.3)$ & & $32(47.8)$ & $134(44.2)$ & \\
\hline Undifferentiated $^{\ddagger}$ & $88(40.7)$ & $404(43.6)$ & & $53(35.6)$ & $235(37.7)$ & & $35(52.2)$ & $169(55.8)$ & \\
\hline Depth of invasion* & & & 0.284 & & & 0.468 & & & 0.412 \\
\hline pT1-T2 & $167(79.1)$ & $643(75.6)$ & & $114(79.2)$ & $435(76.3)$ & & $53(79.1)$ & $208(74.3)$ & \\
\hline pT3-T4 & $44(20.9)$ & $207(24.4)$ & & $30(20.8)$ & $135(23.7)$ & & $14(20.9)$ & $72(25.7)$ & \\
\hline Lymph node & & & 0.197 & & & 0.213 & & & 0.633 \\
\hline \multicolumn{10}{|l|}{ metastasis* } \\
\hline pNO & $117(68.8)$ & $425(63.5)$ & & 78 (69.6) & $280(63.3)$ & & $39(67.2)$ & $145(63.9)$ & \\
\hline pN1-N3 & $53(31.2)$ & $244(36.5)$ & & $34(30.4)$ & $162(36.7)$ & & $19(32.8)$ & $82(36.1)$ & \\
\hline Distant metastasis & & & 0.029 & & & 0.096 & & & 0.140 \\
\hline Absent & $202(93.5)$ & $820(88.5)$ & & 138 (92.6) & $548(87.8)$ & & 64 (95.5) & $272(89.8)$ & \\
\hline Present & $14(6.5)$ & $107(11.5)$ & & $11(7.4)$ & $76(12.2)$ & & $3(4.5)$ & $31(10.2)$ & \\
\hline \multicolumn{10}{|l|}{ TNM stage } \\
\hline I\&II & $181(83.8)$ & 694 (74.9) & 0.005 & 125 (83.9) & 470 (75.3) & 0.026 & 56 (83.6) & 224 (73.9) & 0.096 \\
\hline III\&IV & $35(16.2)$ & $233(25.1)$ & & $24(16.1)$ & $154(24.7)$ & & $11(16.4)$ & $79(26.1)$ & \\
\hline
\end{tabular}


Table 1. Continued

\begin{tabular}{|c|c|c|c|c|c|c|c|c|c|}
\hline & \multicolumn{3}{|c|}{ Total } & \multicolumn{3}{|c|}{ Male } & \multicolumn{3}{|c|}{ Female } \\
\hline & $\begin{array}{c}\mathrm{FH}(+) \\
(\mathrm{n}=216)\end{array}$ & $\begin{array}{l}\text { FH (-) } \\
(\mathrm{n}=927)\end{array}$ & p-value & $\begin{array}{c}\mathrm{FH}(+) \\
(\mathrm{n}=149)\end{array}$ & $\begin{array}{c}\text { FH (-) } \\
(\mathrm{n}=624)\end{array}$ & $\mathrm{p}$-value & $\begin{array}{l}\mathrm{FH}(+) \\
(\mathrm{n}=67)\end{array}$ & $\begin{array}{l}\text { FH (-) } \\
(\mathrm{n}=303)\end{array}$ & $\mathrm{p}$-value \\
\hline TGFB1 C-509T & & & 0.256 & & & 0.633 & & & 0.274 \\
\hline \multicolumn{10}{|l|}{ polymorphism } \\
\hline CC & $63(29.2)$ & $227(24.5)$ & & $40(26.8)$ & $150(24.0)$ & & $23(34.3)$ & $77(25.4)$ & \\
\hline CT & $104(48.1)$ & 500 (53.9) & & $76(51.0)$ & $345(55.3)$ & & $28(41.8)$ & 155 (51.2) & \\
\hline TT & 49 (22.7) & 200 (21.6) & & $33(22.1)$ & $129(20.7)$ & & $16(23.9)$ & $71(23.4)$ & \\
\hline Resection & & & - & & & - & & & - \\
\hline Endoscopic & $56(25.9)$ & $215(23.2)$ & & $43(28.8)$ & $155(24.8)$ & & $13(19.4)$ & $60(19.8)$ & \\
\hline Surgical & $148(68.5)$ & $611(65.9)$ & & $97(65.1)$ & $400(64.1)$ & & $51(76.1)$ & $219(72.2)$ & \\
\hline No & $12(5.6)$ & $101(10.9)$ & & $9(6.1)$ & 69 (11.1) & & $3(4.5)$ & $32(8.0)$ & \\
\hline Follow-up period, mo & $88.96 \pm 43.52$ & $85.66 \pm 48.58$ & & $83.64 \pm 44.44$ & $85.73 \pm 46.54$ & 0.618 & $100.79 \pm 39.24$ & $85.5 \pm 46.75$ & 0.006 \\
\hline Death & $50(23.1)$ & $254(27.4)$ & 0.203 & 41 (27.5) & $174(27.9)$ & 0.928 & $9(13.4)$ & $80(26.4)$ & 0.025 \\
\hline Recurrence & $9(4.2)$ & $30(3.2)$ & 0.498 & $6(4.0)$ & $24(3.8)$ & 0.918 & $3(4.5)$ & $6(2.0)$ & 0.211 \\
\hline
\end{tabular}

Data are presented as mean \pm SD or number $(\%)$.

FH, family history; BMI, body mass index; H. pylori, Helicobacter pylori; pT, tumor depth; pN, lymph node metastasis.

*Patients with incomplete records were excluded; ${ }^{\dagger}$ Includes well- or moderately differentiated adenocarcinoma; ${ }^{ \pm}$Includes poorly differentiated tubular adenocarcinoma, signet ring cell carcinoma, papillary adenocarcinoma, mucinous adenocarcinoma, adenosquamous carcinoma and undifferentiated adenocarcinoma.

Table 2 Comparison of TNM Stages of Gastric Cancer Stratified by a FH and the Presence of the TGFB1 C509T Polymorphism

\begin{tabular}{|c|c|c|c|c|c|c|c|c|c|c|c|c|}
\hline & \multicolumn{4}{|c|}{ Total } & \multicolumn{4}{|c|}{ Male } & \multicolumn{4}{|c|}{ Female } \\
\hline & \multicolumn{2}{|c|}{$\mathrm{FH}(+)$} & \multicolumn{2}{|c|}{ FH (-) } & \multicolumn{2}{|c|}{$\mathrm{FH}(+)$} & \multicolumn{2}{|c|}{$\mathrm{FH}(-)$} & \multicolumn{2}{|c|}{$\mathrm{FH}(+)$} & \multicolumn{2}{|c|}{$\mathrm{FH}(-)$} \\
\hline & $\mathrm{CC}$ & $\mathrm{CC} / \mathrm{TT}$ & $\mathrm{CC}$ & $\mathrm{CC} / \mathrm{TT}$ & $\mathrm{CC}$ & $\mathrm{CC} / \mathrm{TT}$ & $\mathrm{CC}$ & $\mathrm{CC} / \mathrm{TT}$ & $\mathrm{CC}$ & $\mathrm{CC} / \mathrm{TT}$ & $\mathrm{CC}$ & $\mathrm{CC} / \mathrm{TT}$ \\
\hline Stage I\&II & 49 (77.8) & 132 (86.3) & $170(74.9)$ & $524(74.9)$ & $30(75.0)$ & 95 (87.2) & $113(75.3)$ & 357 (75.3) & 19 (82.6) & 37 (84.1) & $57(74.0)$ & 167 (73.9) \\
\hline Stage III\&IV & $14(22.2)$ & 21 (13.7) & $57(25.1)$ & 57 (25.1) & $10(25.0)$ & 14 (12.8) & 37 (24.7) & $117(24.7)$ & $4(17.4)$ & 7 (15.9) & $20(26.0)$ & $59(26.1)$ \\
\hline$p$-value & \multicolumn{2}{|c|}{0.123} & \multicolumn{2}{|c|}{0.992} & \multicolumn{2}{|c|}{0.074} & \multicolumn{2}{|c|}{0.997} & \multicolumn{2}{|c|}{0.876} & \multicolumn{2}{|c|}{0.982} \\
\hline
\end{tabular}

Data are presented as number (\%).

FH, family history.

A

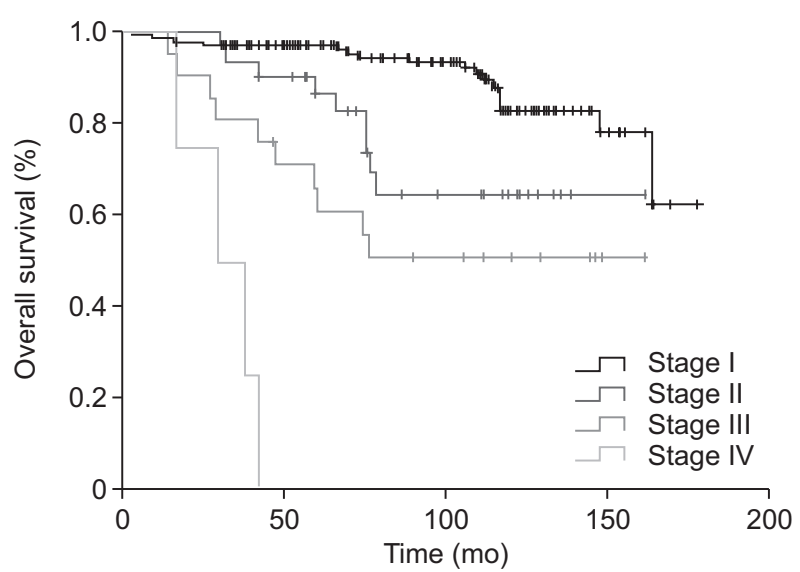

B

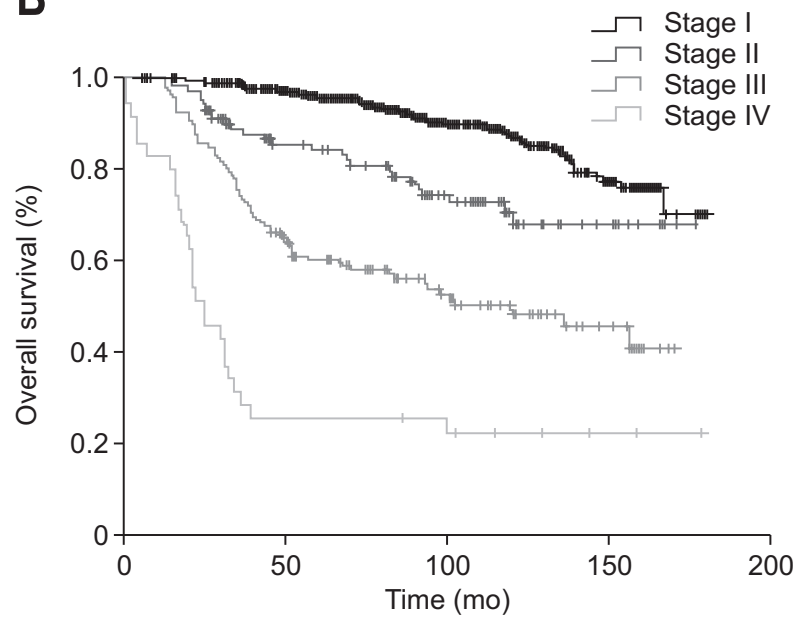

Fig. 1. The overall survival curves according to the stage of gastric cancer in patients with (A) and without a family history of gastric cancer (B). 

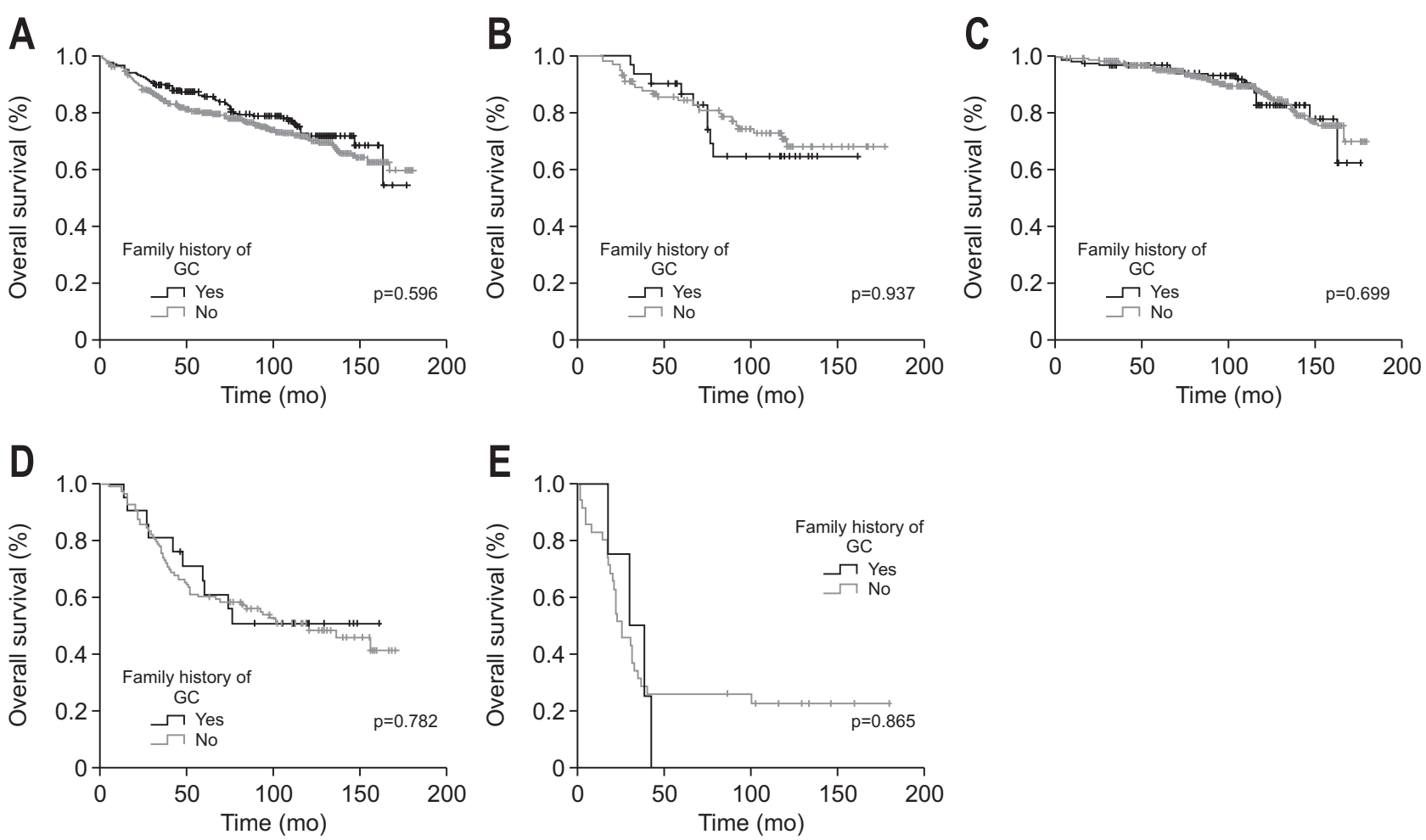

Fig. 2. Comparison of overall survival between patients with and without a family history of gastric cancer (GC) with all stages (A), stage I (B), stages II (C), stage III (D), and stage IV (E).
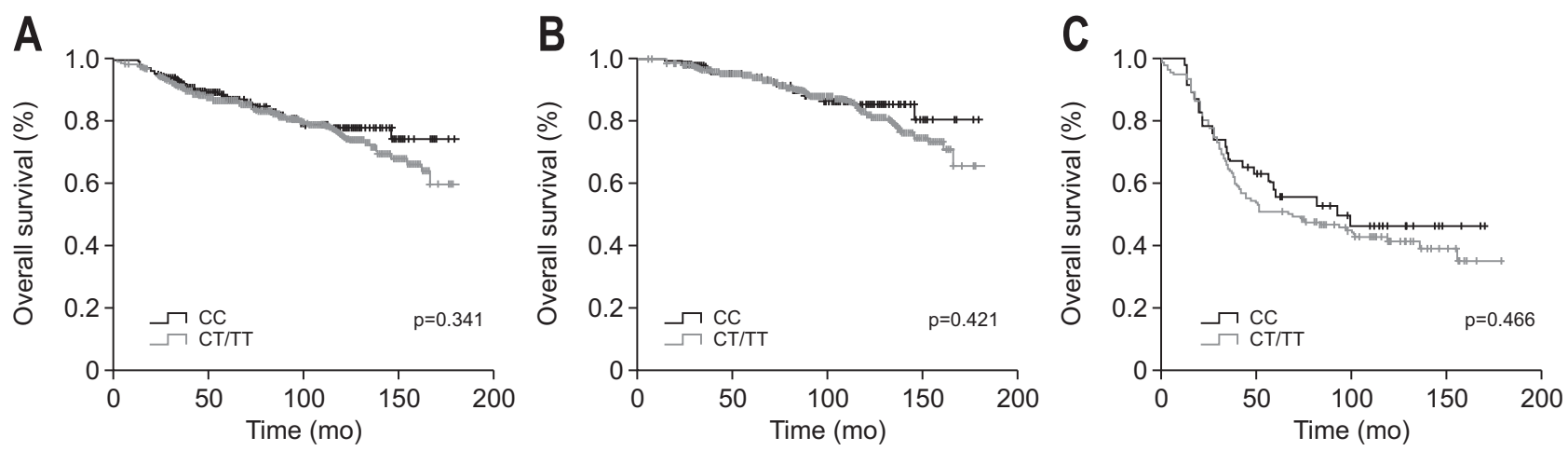

Fig. 3. Comparison of overall survival in patients with the CC and CT/TT genotypes of the TGFB1-509 polymorphism in all stages of gastric cancer (A), stages I-II (B), and stages III-IV (C).

patients with and without a FH (Fig. 4).

Multivariate analyses using Cox proportional hazards model to investigate whether FH of GC and genotypes TGFB1-509 polymorphism affected the overall survival revealed that age, $\mathrm{T}$ stage, $\mathrm{N}$ stage, $\mathrm{M}$ stage, and $H$. pylori infection were independent prognostic factors for overall survival. However, FH of GC and CC genotype were not independent risk factors for overall survival ( $p=0.708$ and $p=0.728$, respectively) (Table 3 ).

\section{DISCUSSION}

We found that patients with a FH had more stage I-II GCs than those without a FH in total and male patient groups; however, except for tumor stage, there were no significant differences in the other characteristics between the patients with and without a FH. The overall survival rates of GC were not significantly different between patients with and without a FH. The association between a FH and stage I-II GC was not significant according to the TGFB1 C-509T polymorphism. Regarding the TGFB1 C-509T polymorphism, tumor stage and overall survival were not different between CT/TT and CC genotypes according to sex and a FH. In addition, FH of GC and CC genotype were not independent risk factors for overall survival.

Previous studies have shown conflicting results on the as- 

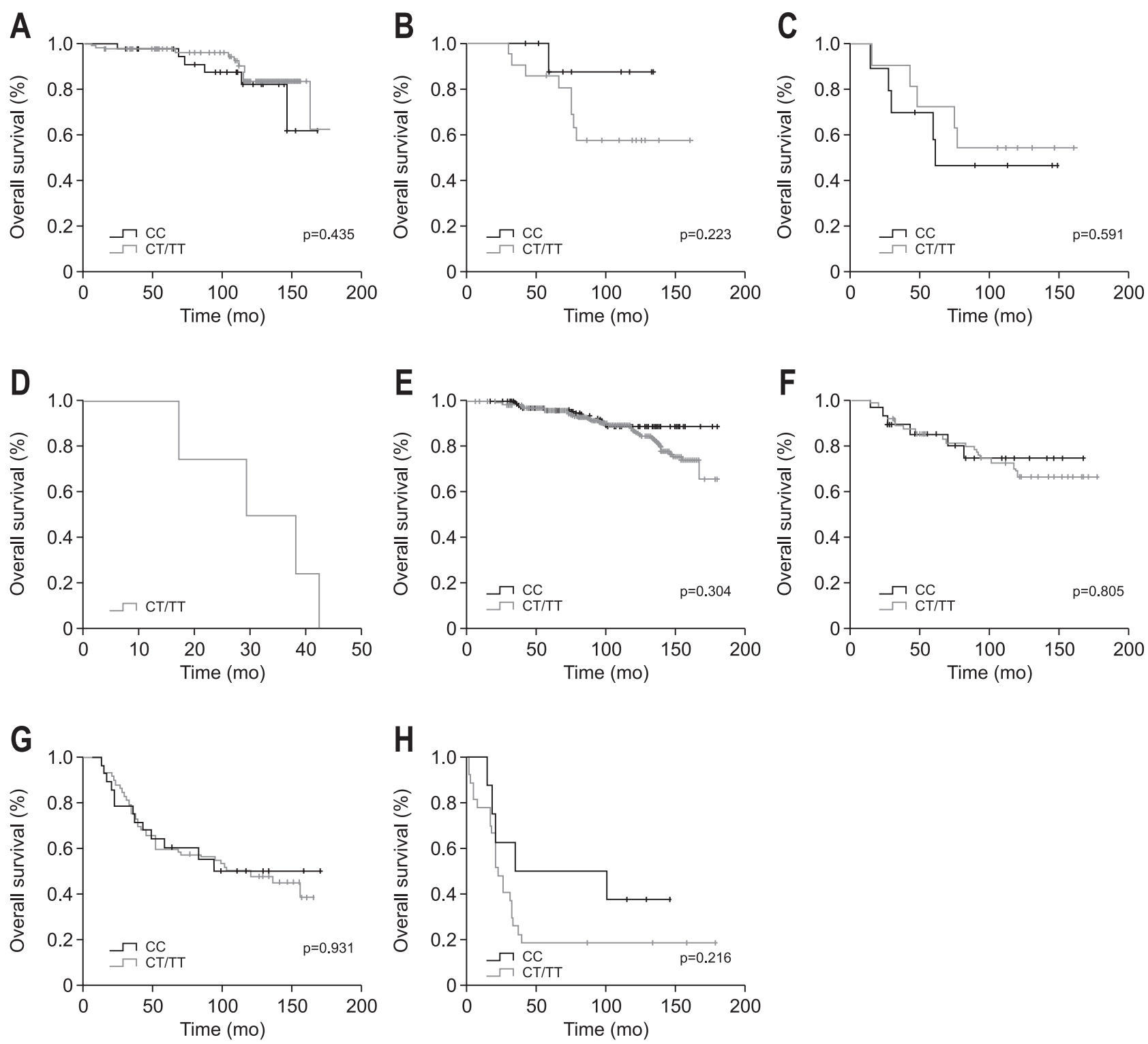

Fig. 4. Comparison of overall survival in patients stratified by family history of gastric cancer and genotypes of TGFB1-509 polymorphism. The overall survival curves of patients with a family history of gastric cancer are shown for those with stage I (A), stage II (B), stage III (C), and stage IV (D). The overall survival curves of patients without a family history of gastric cancer are shown for those with stage I (E), stage II (F), stage III (G), and stage IV (H).

sociation of a FH with GC survival. ${ }^{8-10,24,25}$ A Korean study of 1,273 patients with GC showed that a FH was associated with a reduced risk of recurrence and death in patients with stage IIIIV GC but was limited by the small number of patients in the stage III-IV group (only 48 patients with a $\mathrm{FH}$ ) and the inclusion of 51 patients with second-degree relatives of GC. ${ }^{8}$ By contrast, a recent large Korean study with 2,736 patients reported that the disease-specific survival rate was not significantly different between patients with and without a FH in all stages, but this study also included first- or second-degree relatives with GC. ${ }^{25}$ The strength of the present study is that it is the largest study to investigate the clinicopathological features and survival of patients with only FDRs of GC.
In the present study, patients with a FH were not younger, and patients with a FH did not have a better survival though they had a less advanced tumor stage. In particular, the distant metastasis rate in GC patients with a FH was significantly lower than in those without a FH, and this result might be associated with an earlier diagnosis in patients with a FH due to early and frequent health screening. Korean and Italian studies reported that intestinal-type GCs were more frequent in patients with a $\mathrm{FH}$ than in patients without a $\mathrm{FH},{ }^{8,9}$ which might explain the favorable prognosis of GC patients with a FH. However, our study showed that the proportion of intestinal type GC was not significantly different between patients with and without a FH. The overall survival rates in the female patient group with a FH 
Table 3. Multivariate Analysis of Overall Survival of Patients with Gastric Cancer Using a Cox Proportional Hazards Regression Model

\begin{tabular}{|c|c|c|c|}
\hline Variable & Hazard ratio & $95 \% \mathrm{CI}$ & p-value \\
\hline Age & 1.045 & $1.031-1.059$ & 0.000 \\
\hline Sex (male vs female) & 1.168 & $0.726-1.880$ & 0.523 \\
\hline Smoking (current/ex-smoker vs nonsmoker) & 0.829 & $0.523-1.313$ & 0.424 \\
\hline Drinking (drinker vs nondrinker) & 1.044 & $0.734-1.486$ & 0.810 \\
\hline Body mass index $\left(\geq 25 \mathrm{~kg} / \mathrm{m}^{2} \mathrm{vs}<25 \mathrm{~kg} / \mathrm{m}^{2}\right)$ & 0.836 & $0.578-1.209$ & 0.340 \\
\hline Family history of gastric cancer (positive vs negative) & 0.952 & $0.641-1.415$ & 0.808 \\
\hline H. pylori infection (positive vs negative) & 0.648 & $0.452-0.928$ & 0.018 \\
\hline Lauren histiocyte (diffuse/mixed vs intestinal) & 1.115 & $0.809-1.537$ & 0.504 \\
\hline T stage (T3-T4 vs T1-T2) & 2.196 & $1.498-3.220$ & 0.000 \\
\hline N stage (N1-N3 vs N0) & 2.354 & $1.609-3.444$ & 0.000 \\
\hline M stage (M1 vs M0) & 4.025 & $2.511-6.452$ & 0.000 \\
\hline TGFB1 C-509T polymorphism (CC vs CT+TT) & 1.066 & $0.744-1.527$ & 0.728 \\
\hline
\end{tabular}

CI, confidence interval; H. pylori; Helicobacter pylori.

tended to be better than those without a FH without significant differences. The number of deaths in the female group was only nine (13.4\%). The small number of female patients might be insufficient to prove significance, and the effects of a FH might be significant in a study with a large number of patients.

The molecular pathogenesis associated with GC in patients with a FH of GC has not to be elucidated yet. We hypothesize that genetic differences, such as the TGFB1 polymorphism, might be associated with the prognosis of GC in patients with a FH. Disappointingly, the overall survival was not significantly different according to the TGFB1 C-509T polymorphism. Additionally, the association between a FH and stage I-II GC was not significant according to this polymorphism and sex. Many studies have concluded that $\mathrm{T}$ allele carriers of the TGFB1 C509T polymorphism have an increased risk of GC development. ${ }^{15-18}$ The possibility of using TGFB1 polymorphisms as predictive biomarkers has been studied in other types of cancer. The $\mathrm{T}$ allele carriers of the TGFB1 C-509T polymorphism had a lower 5-year disease-free survival rate in breast cancer, ${ }^{26}$ and the TT genotype of the TGFB1-509 polymorphism was found to be associated with an increased risk of aggressive prostate cancer $^{27}$ and shorter median survival in colorectal cancer. ${ }^{28}$ There have been a few studies on the relationship between the TGFB1 polymorphism and the prognosis of GC. A Chinese case control study reported an association between the C-509T polymorphisms and an increased risk for stage III-IV GC. ${ }^{29}$ Some studies reported that increased TGF- $\beta 1$ expression was associated with poor prognosis of GC. ${ }^{30,31}$ In another study, significantly favorable overall survival was demonstrated in the patients with elevated serum TGF- $\beta 1$ concentrations. ${ }^{32}$ However, these studies were conducted on a small sample size, thus may have been insufficient to lead to significant conclusions. To the best of our knowledge, this is the first study to elucidate the association between the TGFB1 C-509T polymorphism and GC survival.
Multiple causative factors involved in the progression of GC and various polymorphisms affect TGF- $\beta 1$ production. ${ }^{33}$ The functional significance of many TGFB1 polymorphisms remains unclear, and further larger studies are needed to justify such polymorphisms as prognostic biomarkers.

Gastric carcinogenesis might be different according to sex. Male dominance of GC has been well known, ${ }^{23}$ and our group previously reported that the effect of obesity on GC was different between sexes. ${ }^{22}$ The known environmental risk factors between males and females did not explain this difference enough, and a possible role of sex hormones was suggested. Estrogen acts through estrogen receptors (ERs) that have genomic and nongenomic effects. Genomic effects appear through estrogen responsive elements located at the promotor of target genes, and it affects transcription factors such as AP- 1 and Sp- $1 .{ }^{34}$ Nongenomic effects are mediated through protein-kinase cascades. ${ }^{34}$ There has been much evidence on a protective role of estrogen in $\mathrm{GC},{ }^{13}$ which might be mediated through ER $\alpha$ and $\mathrm{ER} \beta{ }^{35,36}$ and some studies have suggested prognostic importance of ER $\beta$ in GC. ${ }^{37,38}$ The roles of estrogen in colorectal cancers were elucidated more clearly, and ER $\beta$ prevents the tumorigenesis of colorectum by regulating mismatch repair gene expression and inducing apoptosis while suppressing the proliferation and inflammatory response. ${ }^{34,39}$ From these results, all analyses were conducted according to sex; however, there were no significant differences between sexes.

In the present study, the mean age at diagnosis of GC in patients with a FH was similar to that in patients without a FH $(60.42 \pm 10.36$ years vs $60.04 \pm 12.42$ years), but this age gap between with and without a FH of GC was not much different compared to other studies. ${ }^{8,40}$ The National Cancer Center in Korea reported that the mean age at diagnosis of GC in patients with a FH was even higher than that for sporadic cancer (57.1 years vs 58.1 years). Fang et $a l^{40}$ showed that patients with a 
FH had a better survival rate as they were younger (65.4 years vs 54.1 years) and had a less advanced tumor stage and that a FH had no direct effect on GC survival; however, the familial GC patients in that study were enrolled on the basis of the strict definition. A lack of awareness of the extent to which GC is familial might exist. The term "familial" often is used as a meaning of only a positive $\mathrm{FH}$, while "hereditary" indicates alterations in specific genes, and a gene defect can be determined in only $1 \%$ to $3 \%$ of cases. There is a possibility that those with inherited GC might be included into general GC with a FH, but this possibility accounts for a very small percentage of familial clustering.

A major limitation of the present study was that a FH of GC was self-reported. However, self-reporting of having a FH with respect to cancer is reasonably accurate, especially for FDRs. ${ }^{41}$ Therefore, any information bias would not likely be significant. The questionnaire covering FH of GC was given to each subject on the day of admission before any definite diagnosis, but it was not given at every visit; thus, there might be subjects who have affected family members with GC after enrollment. In addition, we could not include the data on age at initial endoscopy and intervals of endoscopic screening, which might be different according to a FH.

In conclusion, patients with a FH had lower cancer stage (III) at diagnosis than those without a FH. However, no survival differences between the patients with and without a FH were observed. The TGFB1 C-509T polymorphism did not influence the tumor stage and survival. The influence of a FH on the tumor stage and overall survival was not significant according to the TGFB1 C-509T polymorphism. Although this result was negative, further studies in larger cohorts are needed to clarify the exact role of a FH in GC prognosis.

\section{CONFLICTS OF INTEREST}

No potential conflict of interest relevant to this article was reported.

\section{ACKNOWLEDGEMENTS}

This work was supported by the Seoul National University Bundang Hospital Research fund (grant number: 13-2018-002) and by the National Research Foundation (NRF) of Korea grant for the Global Core Research Center (GCRC) funded by the Korea government (MSIP) (number: 2011-0030001).

\section{AUTHOR CONTRIBUTIONS}

Study concept and design: N.K. Data acquisition: M.K. Data analysis and interpretation: H.J.K. Drafting of the manuscript; critical revision of the manuscript for important intellectual content: H.J.K. Statistical analysis: J.B.L. Obtained funding: N.K.,
S.W. Administrative, technical, or material support; study supervision: N.K.

\section{ORCID}

Hee Jin Kim https://orcid.org/0000-0001-9608-0348 Mingu Kwon Nayoung Kim https://orcid.org/0000-0002-6316-952X https://orcid.org/0000-0002-9397-0406 Jae Bong Lee https://orcid.org/0000-0003-4882-081X Sungho Won https://orcid.org/0000-0001-5751-5089

\section{REFERENCES}

1. Global Burden of Disease Cancer Collaboration, Fitzmaurice C, Allen C, et al. Global, regional, and national cancer incidence, mortality, years of life lost, years lived with disability, and disability-adjusted life-years for 32 cancer groups, 1990 to 2015: a systematic analysis for the Global Burden of Disease Study. JAMA Oncol 2017;3:524-548.

2. Chiba T, Marusawa H, Seno H, Watanabe N. Mechanism for gastric cancer development by Helicobacter pylori infection. J Gastroenterol Hepatol 2008;23(8 Pt 1):1175-1181.

3. Wu AH, Wan P, Bernstein L. A multiethnic population-based study of smoking, alcohol and body size and risk of adenocarcinomas of the stomach and esophagus (United States). Cancer Causes Control 2001;12:721-732.

4. Uemura N, Okamoto S, Yamamoto S, et al. Helicobacter pylori infection and the development of gastric cancer. N Engl J Med 2001;345:784-789.

5. Rivas-Ortiz CI, Lopez-Vidal Y, Arredondo-Hernandez LJR, Castillo-Rojas G. Genetic alterations in gastric cancer associated with Helicobacter pylori infection. Front Med (Lausanne) 2017;4:47.

6. Yaghoobi M, Bijarchi R, Narod SA. Family history and the risk of gastric cancer. Br J Cancer 2010;102:237-242.

7. Oliveira C, Pinheiro H, Figueiredo J, Seruca R, Carneiro F. Familial gastric cancer: genetic susceptibility, pathology, and implications for management. Lancet Oncol 2015;16:e60-e70.

8. Han MA, Oh MG, Choi IJ, et al. Association of family history with cancer recurrence and survival in patients with gastric cancer. $\mathrm{J}$ Clin Oncol 2012;30:701-708.

9. Bernini M, Barbi S, Roviello F, et al. Family history of gastric cancer: a correlation between epidemiologic findings and clinical data. Gastric Cancer 2006;9:9-13.

10. Palli D, Russo A, Saieva C, Salvini S, Amorosi A, Decarli A. Dietary and familial determinants of 10-year survival among patients with gastric carcinoma. Cancer 2000;89:1205-1213.

11. Gao Y, Hu N, Han X, et al. Family history of cancer and risk for esophageal and gastric cancer in Shanxi, China. BMC Cancer 2009;9:269.

12. Oh MG, Kim JH, Han MA, Park J, Ryu SY, Choi SW. Family history and survival of patients with gastric cancer: a meta-analysis. Asian Pac J Cancer Prev 2014;15:3465-3470. 
13. Camargo MC, Goto Y, Zabaleta J, Morgan DR, Correa P, Rabkin CS. Sex hormones, hormonal interventions, and gastric cancer risk: a meta-analysis. Cancer Epidemiol Biomarkers Prev 2012;21:20-38.

14. Elliott RL, Blobe GC. Role of transforming growth factor beta in human cancer. J Clin Oncol 2005;23:2078-2093.

15. Chang WW, Zhang L, Su H, Yao YS. An updated meta-analysis of transforming growth factor-beta1 gene: three polymorphisms with gastric cancer. Tumour Biol 2014;35:2837-2844.

16. Choi YJ, Kim N, Shin A, et al. Influence of TGFB1 C-509T polymorphism on gastric cancer risk associated with TGF-beta1 expression in the gastric mucosa. Gastric Cancer 2015;18:526-537.

17. Li K, Xia F, Zhang K, Mo A, Liu L. Association of a tgf-b1-509c/t polymorphism with gastric cancer risk: a meta-analysis. Ann Hum Genet 2013;77:1-8.

18. Pavithra D, Gautam M, Rama R, et al. TGFbeta C-509T, TGFbeta T869C, XRCC1 Arg194Trp, IKBalpha C642T, IL4 C-590T Genetic polymorphisms combined with socio-economic, lifestyle, diet factors and gastric cancer risk: a case control study in South Indian population. Cancer Epidemiol 2018;53:21-26.

19. Shin CM, Kim N, Lee HS, et al. Intrafamilial aggregation of gastric cancer: a comprehensive approach including environmental factors, Helicobacter pylori virulence, and genetic susceptibility. Eur J Gastroenterol Hepatol 2011;23:411-417.

20. Ebert MP, Yu J, Miehlke S, et al. Expression of transforming growth factor beta-1 in gastric cancer and in the gastric mucosa of first-degree relatives of patients with gastric cancer. $\mathrm{Br} \mathrm{J}$ Cancer 2000;82:1795-1800.

21. Kim HW, Kim JH, Lim BJ, et al. Sex disparity in gastric cancer: female sex is a poor prognostic factor for advanced gastric cancer. Ann Surg Oncol 2016;23:4344-4351.

22. Kim HJ, Kim N, Kim HY, et al. Relationship between body mass index and the risk of early gastric cancer and dysplasia regardless of Helicobacter pylori infection. Gastric Cancer 2015;18:762-773.

23. Freedman ND, Derakhshan MH, Abnet CC, Schatzkin A, Hollenbeck AR, McColl KE. Male predominance of upper gastrointestinal adenocarcinoma cannot be explained by differences in tobacco smoking in men versus women. Eur J Cancer 2010;46:2473-2478.

24. Lee WJ, Hong RL, Lai IR, Chen CN, Lee PH, Huang MT. Clinicopathologic characteristics and prognoses of gastric cancer in patients with a positive familial history of cancer. J Clin Gastroenterol 2003;36:30-33.

25. Jeong O, Jung MR, Park YK, Ryu SY. Clinicopathological features and survival of patients with gastric cancer with a family history: a large analysis of 2,736 patients with gastric cancer. J Gastric Cancer 2017;17:162-172.

26. Shu XO, Gao YT, Cai Q, et al. Genetic polymorphisms in the TGFbeta 1 gene and breast cancer survival: a report from the Shanghai Breast Cancer Study. Cancer Res 2004;64:836-839.

27. Ewart-Toland A, Chan JM, Yuan J, Balmain A, Ma J. A gain of function TGFB1 polymorphism may be associated with late stage prostate cancer. Cancer Epidemiol Biomarkers Prev 2004;13:759764.

28. Gulubova M, Aleksandrova E, Vlaykova T. Promoter polymorphisms in TGFB1 and IL10 genes influence tumor dendritic cells infiltration, development and prognosis of colorectal cancer. J Gene Med 2018;20:e3005.

29. Zhang P, Di JZ, Zhu ZZ, et al. Association of transforming growth factor-beta 1 polymorphisms with genetic susceptibility to TNM stage I or II gastric cancer. Jpn J Clin Oncol 2008;38:861-866.

30. Vagenas K, Spyropoulos C, Gavala V, Tsamandas AC. TGFbeta1, TGFbeta2, and TGFbeta3 protein expression in gastric carcinomas: correlation with prognostics factors and patient survival. J Surg Res 2007;139:182-188.

31. Hawinkels LJ, Verspaget HW, van Duijn W, et al. Tissue level, activation and cellular localisation of TGF-beta1 and association with survival in gastric cancer patients. Br J Cancer 2007;97:398404.

32. Park YS, Park DJ, Lee Y, et al. Prognostic roles of perioperative body mass index and weight loss in the long-term survival of gastric cancer patients. Cancer Epidemiol Biomarkers Prev 2018;27:955-962.

33. Martelossi Cebinelli GC, Paiva Trugilo K, Badaró Garcia S, Brajão de Oliveira K. TGF-beta1 functional polymorphisms: a review. Eur Cytokine Netw 2016;27:81-89.

34. Nie X, Xie R, Tuo B. Effects of estrogen on the gastrointestinal tract. dig dis sci 2018;63:583-596.

35. Jukic Z, Radulovic P, Stojković R, et al. Gender difference in distribution of estrogen and androgen receptors in intestinal-type gastric cancer. Anticancer Res 2017;37:197-202.

36. Ryu WS, Kim JH, Jang YJ, et al. Expression of estrogen receptors in gastric cancer and their clinical significance. J Surg Oncol 2012;106:456-461.

37. Wang M, Pan JY, Song GR, Chen HB, An LJ, Qu SX. Altered expression of estrogen receptor alpha and beta in advanced gastric adenocarcinoma: correlation with prothymosin alpha and clinicopathological parameters. Eur J Surg Oncol 2007;33:195-201.

38. Xu CY, Guo JL, Jiang ZN, et al. Prognostic role of estrogen receptor alpha and estrogen receptor beta in gastric cancer. Ann Surg Oncol 2010;17:2503-2509.

39. Kim SE, Paik HY, Yoon H, Lee JE, Kim N, Sung MK. Sex- and gender-specific disparities in colorectal cancer risk. World J Gastroenterol 2015;21:5167-5175.

40. Fang WL, Chang SC, Lan YT, et al. Molecular and survival differences between familial and sporadic gastric cancers. Biomed Res Int 2013;2013:396272.

41. Kerber RA, Slattery ML. Comparison of self-reported and database-linked family history of cancer data in a case-control study. Am J Epidemiol 1997;146:244-248. 